\title{
Search for GRB afterglows in the ROSAT all-sky survey
}

\author{
J. Greiner ${ }^{1}$, W. Voges ${ }^{2}$, T. Boller ${ }^{2}$, and D. Hartmann ${ }^{3}$ \\ 1 Astrophysical Institute Potsdam, An der Sternwarte 16, 14482 Potsdam, Germany \\ 2 MPI for extraterrestrial Physics, 85740 Garching, Germany \\ 3 Clemson Univ., Dept. of Phys and Astronomy, Clemson, SC 29634-1911, U.S.A.
}

Received December 22, 1998; accepted May 10, 1999

Abstract. We report on the status of our search for X-ray afterglows of gamma-ray bursts (GRBs) using the ROSAT all-sky survey (RASS) data. The number of potential $\mathrm{X}$-ray afterglow candidates with respect to the expected number of beamed GRBs allows to constrain the relative beaming angles of GRB emission and afterglow emission at about $1-5$ hrs after the GRB.

Key words: gamma-rays: bursts — X-rays: general

\section{Introduction}

The recent discovery of long-lasting X-ray afterglow emission from (at least some) gamma-ray burst (GRB) sources has allowed the first identification of these enigmatic objects outside the gamma-ray range. Optical observations of these GRB counterparts suggest a cosmological distance scale. According to the standard scenario, the beaming angle increases with decreasing photon energy (Meszaros \& Rees 1997). Though it is yet unclear what fraction of the X-ray emission is part of the GRB itself or indeed the $\mathrm{X}$-ray afterglow produced by the deceleration of the fireball shock wave by the interstellar medium, one expects that the beaming to be narrower in the $\gamma$ ray range, simply because $\gamma$ 's come from more relativistic electrons and thus the $1 / \Gamma$ factor leads to more forward beaming. This implies that the rate of X-ray (and other long-wavelengths) afterglows should be considerably higher than the GRB rate. A systematic search for afterglows therefore allows to constrain the beaming geometry of GRB emission (Rhoads 1997), in that the ratio of afterglows vs. GRBs determines the geometric beaming factor of the GRB emission.

\section{ROSAT all-sky survey data and expectations}

The ROSAT satellite performed the first all-sky survey in the $0.1-2.4 \mathrm{keV}$ band during 1990 August $1-1991$ January 25 with short additional exposures ("repairs") in February 16-18 and August 4-12, 1991. During the satellites orbital period of 96 min the telescope with a field of view of nearly $2^{\circ}$ diameter scans a full $360^{\circ}$ circle on the sky. Thus, the exposure per scan on a given sky location is between $10-30 \mathrm{~s}$. Due to the orbital plane rotation (together with Earth's motion) these full circles move with $1^{\circ} /$ day perpendicular to the scan direction, covering the

Send offprint requests to: J. Greiner, jgreiner@aip.de whole sky in 6 months. Thus, a sky location at the ecliptic equator is covered by the telescope scans over two days, and this coverage rises to 180 days at the ecliptic poles. Similarly, the typical sky exposure is a function of ecliptic latitude and amounts to $200 \mathrm{~s}$ at the equator and up to $40 \mathrm{ksec}$ at the poles.

ROSAT is sensitive enough to detect a GRB X-ray afterglow for a few hours within its $10-30 \mathrm{~s}$ exposure time per sky location per scan. Figure 1 shows the one-scan sensitivity of ROSAT relative to the measured $\mathrm{X}$-ray afterglow decay curves. The fraction $f$ of afterglows detectable during the RASS depends on three critical parameters. First, the fraction of GRBs displaying X-ray afterglows: Previous observations suggest this fraction to be close to one. Second, the possible relation of X-ray flux to $\gamma$-ray peak flux or fluence: So far, the observed X-ray afterglow fluxes at about $100 \mathrm{~s}$ after the GRB are spread within a factor of 10 only, while the GRB fluxes range over a factor of $>1000$. Third, the slope of the X-ray intensity decay: observed values range between $t^{-1.8} \ldots t^{-2.5}$. The effect of the combination of the latter two factors is difficult to assess in an accurate manner given the low statistics at present, so we base our estimate of $f$ on the observed X-ray afterglow intensities. A comparison with the ROSAT PSPC sensitivity suggests that we would detect practically all GRB afterglows in 3 subsequent scans, and $\sim 80 \%$ in 5 scans (see Fig. 1). Thus, we conservatively adopt $f=0.8$ in the following.

The number of detectable X-ray afterglows from a GRB beamed towards us (based on the BATSE detection rate) during the RASS is

$f \times S_{\mathrm{R}}^{\mathrm{agl}} \times R_{\mathrm{GRB}}$

where $R_{\mathrm{GRB}}$ is the rate of beamed GRBs per time and area on the sky and $S_{\mathrm{R}}^{\mathrm{agl}}$ is the effectiveness of ROSAT for afterglows in units of time $\times$ area. We adopt $R_{\mathrm{GRB}}=$ $900 \mathrm{GRBs} / \mathrm{sky} / \mathrm{yr}=1 \mathrm{GRB} /\left(16628 \square^{\circ} \times\right.$ days $) . S_{\mathrm{R}}^{\mathrm{agl}}$ would be $122296.5 \square^{\circ} \times$ days for a $100 \%$ perfect survey. With a temporal completeness of the RASS of $62.5 \%$ we use $S_{\mathrm{R}}^{\mathrm{agl}}$ $=76435 \square^{\circ} \times$ days in the following. Thus, we would expect $4.6 \times f$ afterglows of beamed GRBs to be detected during the RASS. 


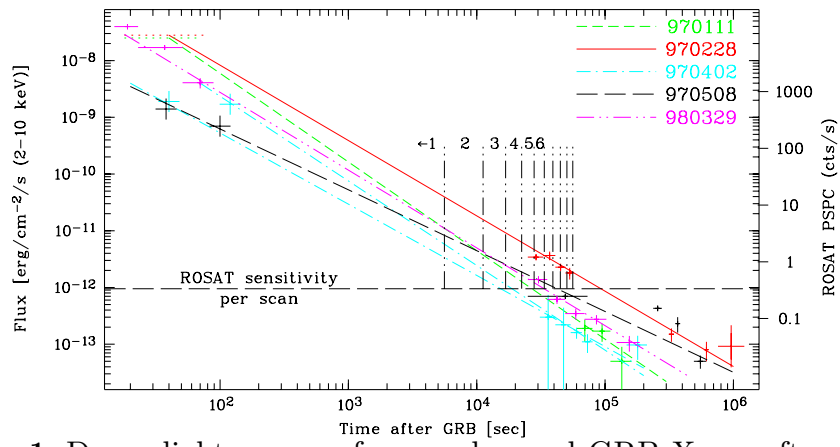

Fig. 1. Decay light curves of some observed GRB X-ray afterglows in the 2-10 keV range (GRB 970111: Feroci et al. 1998; GRB 970228: Costa et al. 1997; GRB 970402: Nicastro et al. 1998; GRB 970508: Piro et al. 1998; GRB 980329: in 't Zand et al. 1998) and their corresponding brightness extrapolated into the ROSAT band (scale on the right; assuming a power law with photon index of -2 and no absorption). The horizontal line gives the sensitivity of the ROSAT PSPC during one scan, and the vertical lines mark the time windows for the possible coverage of a GRB location by ROSAT during its scanning mode. Thus, one may expect an afterglow at an intensity of up to several hundred cts/s during the first scan, between $0.3-8 \mathrm{cts} / \mathrm{s}$ during the second scan, $<2 \mathrm{cts} / \mathrm{s}$ during the third scan and so on

\section{Data selection and results}

We have first produced scan-to-scan light curves for all RASS sources with either a count rate larger than $0.05 \mathrm{cts} / \mathrm{s}$ or a detection likelihood larger than 10 , resulting in a total of 25176 light curves. (Note that these criteria correspond to a lower sensitivity threshold as compared to the RASS Bright Source Catalog which invoked at least 15 counts and a detection likelihood $\gtrsim 15$ ). Each of these light curves consists of about 20450 bins spaced at $96 \mathrm{~min}$, with each bin corresponding to $10-30 \mathrm{~s}$ exposure time.

After ignoring 363 light curves with negative mean count rates (caused by incorrect background-subtraction in our automatic procedure) we apply two conditions to these light curves: (1) the maximum bin should have a signal-to-noise ratio $S / N>3$ above the mean count rate around the maximum $(S / N$ is defined as difference between the maximum and mean count rate divided by the square root of the quadratic sum of the error of the maximum and mean count rates, respectively), and (2) the mean count rate until one bin before the maximum as well as the mean count rate for times later than 5 bins after the maximum should be consistent with zero. This yields 32 candidate sources. We then excluded visually (i) sources with double and multipeak structures (4), and (ii) sources with a rise over $2-3$ bins and zero flux immediately after the maximum, e.g. like an inverted decay (3). In addition, we investigated the available pointed observations for 3 of these candidates, and excluded all 3 sources because we find persistent X-ray emission at a level below the RASS threshold. Finally, we made a correlation with various optical, infrared and radio catalogs and excluded sources with known counterparts as well as sources with likely counterparts (3). After this procedure we are left with 19 GRB afterglow candidates.

\section{Discussion}

Half of these remaining light curves are single peak events, i.e. have just one bin with $S / N>3$ and otherwise zero count rate. These events could be either statistical fluctuations, flare stars, or GRB afterglows with a very fast decay slope. We note that these single peak events cannot be distinguished from late-type flare stars other than by optical follow-up investigations, since typical time scales of flares range between $10-60 \mathrm{~min}$, just as we observe. We therefore divide our conclusion into two cases:

1. If the single peak events turn out to be due predominantly to flare stars, then the remaining 9 multipeak events (and light curves consistent with a power-law decay) found versus 4 events expected is at first order consistent with the BeppoSAX picture and implies that beaming may not be required.

2. If the single peak events are GRB afterglows then geometric beaming of the GRB emission is possible, but at a moderate value.

In order to more accurately determine the fraction $f$ and thus the expected number of events, a distribution function of power-law indices of GRB X-ray afterglow decays as measured by BeppoSAX is highly needed.

We finally note that GRBs from directions within a few degrees of the galactic plane will suffer absorption, thus compromising the sensitivity of the ROSAT detection. However, this effect concerns only a few percent of the sky, and mainly applies to single peak events, because any multi-peak event should have a first peak count rate high enough to still be detectable.

Acknowledgements. We are indebted to E. Costa and J. in 't Zand for providing X-ray afterglow light curves in digital form. JG is supported by the German Bundesministerium für Bildung, Wissenschaft, Forschung und Technologie (BMBF/DLR) under contract No. 50 OR 9609 8. The ROSAT project is supported by BMBF/DLR and the Max-PlanckSociety. This research has made use of the Simbad database, operated at CDS, Strasbourg, France and the Digitized Sky Survey (DSS) produced at the Space Telescope Science Institute under US Government grant NAG W-2166.

\section{References}

Costa E., Frontera F., Heise J., et al., 1997, Nat 387, 783

Feroci M., Antonelli L.A., Guainazzi M., et al., 1998, A\&A 332, L29

in 't Zand J.J.M., Amati L., Antonelli L.A., et al., 1998, ApJ 505, L119

Meszaros P., Rees M.J., 1997, ApJ 476, 232

Nicastro L., Amati L., Antonelli L.A., et al., 1998, A\&A 338, L17

Piro L., Amati L., Antonelli L.A., et al., 1998, A\&A 331, L41 Rhoads J.E., 1997, ApJ 487, L1 